\title{
Bart's Syndrome: A Neonatal Observation about a Case Report
}

\author{
Assetou Cissouma ${ }^{1 *}$, Djibril Kassogue ${ }^{2}$, Mala Sylla ${ }^{3}$, Guedioma Dembélé4, \\ Soumaila Alama Traoré1, Abdoulaye Kissima-Traoré1, Dadé Ben Sidi Haidara ${ }^{1}$, \\ Mamadou Bernard Coulibaly¹, Madou Traore'1, Mady Niakaté ${ }^{5}$
}

${ }^{1}$ Sikasso Hospital, Sikasso Region, Mali

${ }^{2}$ Timbuktu Hospital, Timbuktu Region, Mali

${ }^{3}$ Bougouni Reference Health Centre, Bougouni, Mali

${ }^{4}$ Mali Hospital, Bamako, Mali

${ }^{5}$ Luxembourg Hospital, Bamako, Mali

Email: *cis_astou@yahoo.fr

How to cite this paper: Cissouma, A., Kassogue, D., Sylla, M., Dembélé, G., Traoré, S.A., Kissima-Traoré, A., Haidara, D.B.S., Coulibaly, M.B., Traore, M. and Niakaté, M. (2021) Bart's Syndrome: A Neonatal Observation about a Case Report. Open Journal of Pediatrics, 11, 406-412.

https://doi.org/10.4236/ojped.2021.113038

Received: July 17, 2021

Accepted: August 31, 2021

Published: September 3, 2021

Copyright $\odot 2021$ by author(s) and Scientific Research Publishing Inc. This work is licensed under the Creative Commons Attribution International License (CC BY 4.0).

http://creativecommons.org/licenses/by/4.0/

(c) (i) Open Access

\begin{abstract}
Introduction: Bart's syndrome is a rare neonatal pathology combining congenital skin aplasia affecting the extremities and congenital epidermolysis bullosa, exceptionally described on black skin. Observation: A 2-day-old male newborn was referred for multiple ulcerations of the limbs observed at birth. The clinical examination found an absence of bilateral and symmetrical skin occupying almost all of the two lower limbs with some flaccid bubbles. The vascular network was clearly visible. The rest of the skin coating was normal. The diagnosis of Bart syndrome in connection with epidermolysis bullosa was evoked clinically and despite pediatric and dermatological management, the evolution was rapidly fatal by severe sepsis. Discussion: Bart syndrome corresponds to a clinical picture of congenital skin aplasia associated with congenital epidermolysis bullosa suspected by areas of fragility and sometimes bubbles. All types of congenital epidermolysis bullosa may be associated with this syndrome. The clinical diagnosis is generally easy but the therapeutic management is difficult and the prognosis reserved. Conclusion: Bart syndrome is a curious congenital association of well-defined skin symptoms, the etiopathogeny of which still remains poorly elucidated, hence the difficulty of establishing an antenatal diagnostic strategy or giving appropriate genetic advice.
\end{abstract}

\section{Keywords}

Skin Aplasia, Congenital, Newborn, Skin Black 


\section{Introduction}

Bart syndrome is a rare neonatal pathology combining congenital skin aplasia affecting the extremities and exceptionally described congenital epidermolysis bullosa on black skin, as well as ungeal deformities. Clinical diagnosis is easy but its management is delicate and the extended forms represent a real therapeutic challenge. It was first described in a family by Bart et al. in 1966 [1]. Pathophysiology is still poorly understood. The goal of management is to prevent secondary complications and to prepare the ground for possible reconstructive surgery. The prognosis is generally good and depends directly on the proper management of skin lesions [2]. About twenty forms of congenital and hereditary epidermolysis bullosa are observed and are classified into three groups according to the level where cleavage occurs: in the dermorepidermal junction zone: these are the simple and non-scarring inepidermal forms, at the level of the pars lucida: these are the junctional forms and under the basal lama: these are the dystrophic forms. We report an observation of a neonatal Bart syndrome on black skin, and propose a review of the literature.

\section{Observation}

He is a male newborn, born to apparently healthy, first-degree consanguineous parents, a primigestprimipare mother who has had a poorly followed pregnancy to term, and a vaginal delivery. She was admitted on the second day of life on suspicion of early neonatal infection from the regional referral health center where he was born. The infectious anamnesis was negative. The newborn had been presenting skin lesions since birth, with the parents finding at $\mathrm{d} 2$ of life of an unquantified fever. The entrance examination found a term newborn, hytrophic, with a weight of $1760 \mathrm{~g}$ (less than-2 DS of the reference curves), a height of 46 $\mathrm{cm}$ (in the average), a head circumference of $33 \mathrm{~cm}$ (in the average). The hemodynamic state was stable, with a fever at $38.6^{\circ} \mathrm{C}$, a skin recoloration time of less than three seconds, a normal heart rate of 160 beats/min, and a respiratory rate of 40 cycles/min. minimal respiratory distress with $97 \%$ oxygen saturation. There were post-bullous erosions at the outer face of the leg and right foot (Figure 1), an absence of skin extending from the back of the foot to the middle $1 / 3$ of the thigh. The lesions were bilateral and symmetrical occupying almost all of both legs and the front faces of the thighs only a few islands of healthy skin persisted on both legs (Figure 2). This absence of skin was also noted on the outer side of the left and right hands with skin fragility and some flaccid bubbles (Figure 3). The edges of the lesion were sharp and the skin on the periphery had a normal color. The vascular network was clearly visible at the level of substance loss. The rest of the skin coating was normal. The integuments and mucous membranes were unremarkable. The nails were dystrophic. In front of this atypical clinical picture associating a lack of localized skin predominant in the lower limbs and a skin fragility, the histopathological examination in the bullous zone allowed to show a dermoepidermal cleavage evocating a bullous epidermolysis 


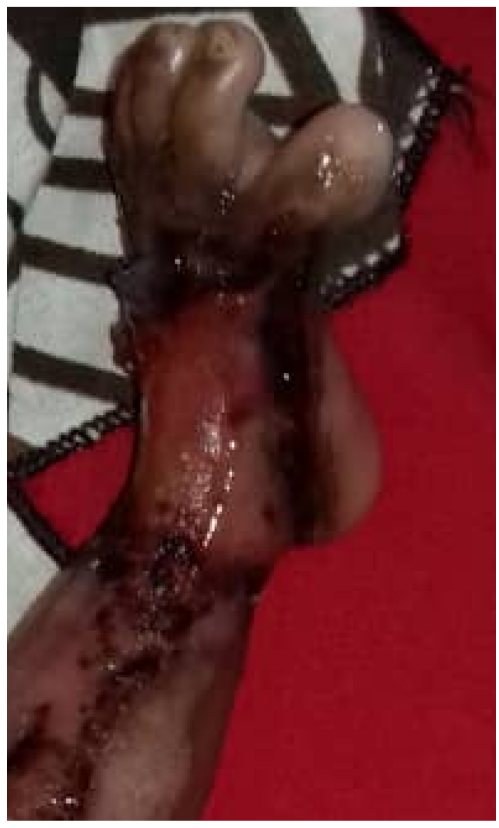

Figure 1. Ruptured bubbles on the right foot.

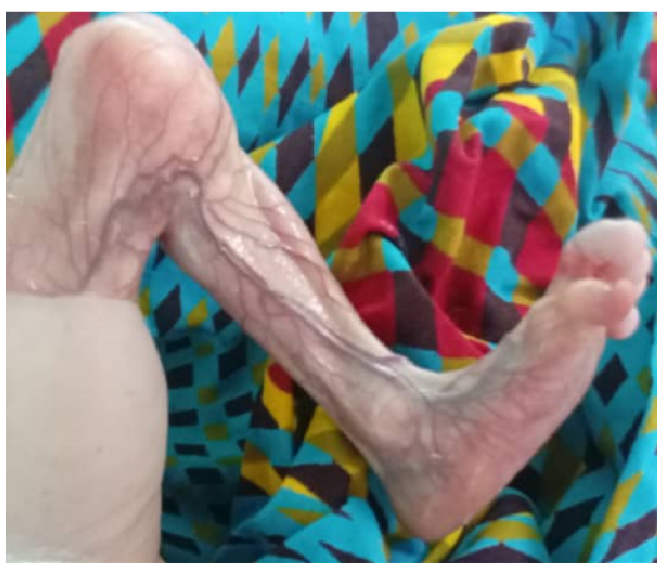

Figure 2. Skin aplasia of the left leg and foot.

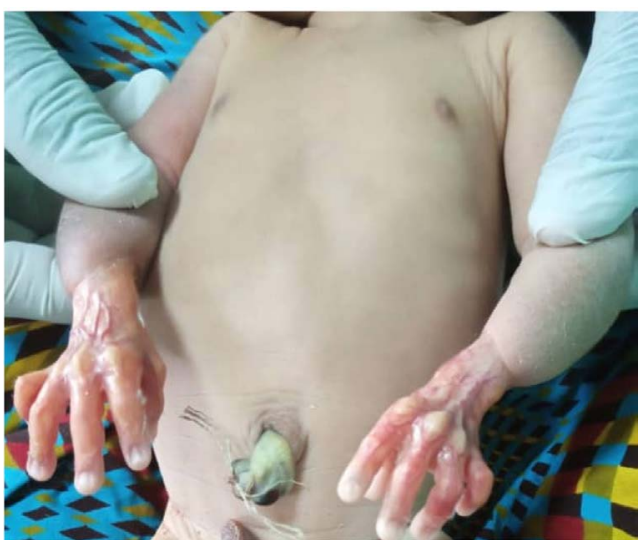

Figure 3. Skin aplasia of the hands (left and right) with the presence of small bubbles. 
of junctional type. The blood test is detailed in Table 1. The diagnosis of Bart syndrome was retained. Hydration of the child to compensate for water loss, antibiotic coverage with third-generation cephalosporins and intravenous gentamicin. Conservative treatment consisted of twice-daily local care and coverage of all lesions by vaseline compresses with gentle manipulation and analgesic treatment. The child died five days later despite this therapeutic management in a severe sepsis table. Parents were referred for genetic counseling.

\section{Discussion}

Bart syndrome is characterized by a clinical triad: congenital cutaneous aplasia (ACC) localized at the extremities, congenital epidermolysis bullosa (EBC) and occasional ungeal deformities [1] [3] [4]. Congenital skin aplasia is a rare dermatosis, it is defined as the localized absence of the skin at birth [5]. It is most often sporadic, but familial cases have been reported [6]. This congenital defect remains generally limited to the vertex in $85 \%$ of cases. The involvement of the limbs is generally bilateral and symmetrical but not very extensive unlike our case. His pathophysiology is very controversial [5] [6] [7]. Some authors explain it by intrauterine mechanical trauma, in particular by friction. This idea is however debated since the lesions of Bart syndrome are generally bilateral and more or less symmetrical. Also, their localization at the level of the plants of the feet and toes makes it difficult to theory of trauma by intrauterine friction. Other authors have made the link between the topography of ACC lesions and Blaschko lines, cutaneous embryonic migration lines that take, among other things, an italic S arrangement along the limbs. ACC lesions that follow these lines are symmetrical, and have a characteristic appearance of S-bands with well-delineated edges. Finally, adhesion of amniotic bands to the skin, placental infarction or death of a twin fetus in utero have also been proposed [5]. Spontaneous healing in the form of an atrophic scar sometimes intrauterine is possible. Extended and deadly forms are rare. Aplasia is sometimes associated with minor malformations or polymalformative syndromes. Clinically our patient had no other malformations. EBC are genodermatoses corresponding to an attack on the dermoepidermal junction, responsible for the formation of bubbles and dermal erosions [8]. There are three types depending on the level of dermoepidermal cleavage: simple epidermolysis bullosa (EBS), junctional EB (EBJ) and dystrophic EB (EBD). We distinguish EBS where the cleavage is intraepidermal in the epidermal

Table 1. Blood test results.

\begin{tabular}{cc}
\hline Blood tests & Result \\
\hline C-reactive protein $(\mathrm{CRP})$ & $150 \mathrm{mg} / \mathrm{L}$ \\
Hemogram & normocytic anemia normochrome at $12 \mathrm{~g} / \mathrm{dl}$ \\
rhesus group & 0 positive \\
glycemia & normal \\
syphilitic serology & negative \\
\hline
\end{tabular}


basal layer, EBJ where the cleavage is located at the level of the basement membrane itself, and EBD where the cleavage is located under the basement membrane, more precisely at the level of the anchoring fibrils of the superficial dermis. There are several differential diagnoses to be eliminated before retaining $\mathrm{CBC}$, mainly infectious causes.

Bart syndrome is increasingly considered an intrauterine variant of EBD [6] [9] [10]. This is due to the fact that the mutation of the p21 region of the short arm of chromosome 3, involved in Bart's syndrome, is contiguous to the type VII collagen gene (COL7A1) which encodes for type 7 collagen fibers, the main constituent of anchoring fibrils allowing cohesion between the dermis and the basement membrane [11] [12]. The mutation of the COL7A1 gene, which results in a substitution of glycine into arginine, is the main mutation of EBD [8]. That being said, since Bart syndrome can be found in any type of EBC [13] [14] [15] as in the case of our patient where Bart syndrome was associated with EBJ, several authors consider that Bart syndrome is only the result of a simultaneous intrauterine presence of a localized absence of the skin and EBC [12]. All in all, despite the still poorly understood genetic basis of Bart syndrome, it is nonetheless considered to be an autosomal dominant genetic disease with complete penetrance and variable expression [11]. The ungeal malformations encountered during this syndrome are ungeal dystrophy, onychomadesis, and anonychia [6] [16]. The mechanism of ungeal involvement in the context of Bart syndrome is so far poorly elucidated. It is an exceptionally reported syndrome in black newborns.

The treatment is first conservative, aimed at promoting the epithelialization and therefore the healing of skin lesions, then possibly surgical for restorative purposes [5] [8] [17]. Conservative treatment relies mainly on local care, by applying non-sticky vaseline gauze dressings to clean erosions and antibiotic ointments to superinfected lesions. A bath is recommended every day or every other day, using diluted antiseptics, also used to remove dressings in the bath if they stick too much. Bubbles should be pierced, and handling should be as gentle as possible to avoid further skin trauma. To prevent syndactyly, small bands between the fingers and toes are recommended. In the case of sepsis with a cutaneous starting point as in the case of our patient, intravenous antibiotic therapy first probabilistic and then oriented by the antibiogram is indicated. Analgesic treatment is a very important part of conservative treatment, since local care is very painful, but unfortunately unavoidable. Complications that can be grafted during conservative treatment are infections (secondary sepsis, localized superinfection), hemorrhage due to skin fragility, dehydration and hydroelectrolytic disorders, hypothermia, and subsequently, atrophic or hypertrophic scars [6] [8] [18]. On chronic erosions that do not heal, autografts or skin allografts may be used. The prognosis is generally favourable, the earlier the management is better, and the less severe the CTE [6] [19] [20].

Genetic counseling is very difficult given the highly variable mode of expres- 
sion of the disease as well as the interindividual and interfamilial difference of the phenotype.

\section{Conclusion}

Bart syndrome is a curious congenital association of well-defined skin symptoms namely ACC, EBC and ungeal involvement, the etiopathogeny of which is still poorly elucidated, hence the difficulty of establishing an antenatal diagnosis strategy or giving appropriate genetic advice. If the clinical diagnosis is easy, the management is complex aimed at healing the skin lesions as well as possible while avoiding secondary complications, thus directly conditioning the prognosis.

\section{Conflicts of Interest}

The authors do not declare any conflict of interest.

\section{Parental Consent}

Informed consent from the parents was obtained for the publication.

\section{Contributions by Authors}

All the authors contributed to the realization of this work. All of them read and approved the final version of the manuscript.

\section{References}

[1] Bart, B.J., Gorlin, R.J. and Anderson, E.V. (1966) Congenital Localized Absence of Skin and Associated Abnormalities Resembling Epidermolysis Bullosa. A New Syndrome. Archives of Dermatology, 93, 296-304. https://doi.org/10.1001/archderm.1966.01600210032005

[2] Benvenuto, C., Kraemer, C.K. and Kruse, R.L. (2003) Familial Epidermolysis Bullosa with Aplasia Cutis Congenita: Bart's Syndrome? Skinmed, 2, 319-321. https://doi.org/10.1111/j.1540-9740.2003.02056.x

[3] Bart, B.J. (1970) Epidermolysis Bullosa and Congenital Localized Absence of Skin. Archives of Dermatology, 101, 78-81. https://doi.org/10.1001/archderm.1970.04000010080014

[4] Gaikwad, A.K., Shende, R. and Khedkar, M.Y. (1993) Bart Syndrome. Indian Journal of Dermatology, Venereology and Leprology, 59, 151-153.

[5] Grosshans, E. (1999) Malformations congénitales de la peau. EMC Dermatologie, 59,10 .

[6] Aparecida Pereira de Almeida, N., Serafini, F. and Marchiori, J. (2010) Do You Know This Syndrome? Anais Brasileiros de Dermatologia, 85, 951-953. https://doi.org/10.1590/S0365-05962010000100023

[7] Frieden, I.J. (1986) Aplasia cutis congenita: A clinical review and proposal for classification. Journal of the American Academy of Dermatology, 14, 646-660. https://doi.org/10.1016/S0190-9622(86)70082-0

[8] Ingen-Housz-Oro, S. and Blanchet-Bardon, C. (2004) Épidermolyses bulleuses héréditaires. EMC Dermatologie, 11, 98-255-A-1. 
https://doi.org/10.1016/j.emcdc.2003.12.004

[9] Aygun, A.D., Yilmaz, E. and Kurt, A.N. (2010) Aplasia cutis congenita and epidemolysis bullosa: Bart syndrome. International Journal of Dermatology, 49, 343-345. https://doi.org/10.1111/j.1365-4632.2009.04182.x

[10] Gutzmer, R., Herbst, R.A. and Becker, J. (1997) Bart Syndrome-Separate Entity or a Variant of Epidermolysis Bullosa? Hautarzt, 48, 640-644. https://doi.org/10.1007/s001050050637

[11] Zelickson, B., Matsumura, K. and Kist, D. (1995) Bart's Syndrome. Ultrastructure and Genetic Linkage. Archives of Dermatology, 131, 663-668.

https://doi.org/10.1001/archderm.1995.01690180037006

[12] Christiano, A.M., Bart, B.J. and Epstein Jr., E.H. (1996) Genetic Basis of Bart's Syndrome: A Glycine Substitution Mutation in Type VII Collagen Gene. Journal of Investigative Dermatology, 106, 778-780.

https://doi.org/10.1111/1523-1747.ep12346304

[13] Fine, J.D., Pulkkinen, L. and Uitto, J. (2000) Congenital Pyloric Atresia in a Newborn with Extensive Aplasia Cutis Congenita and Epidermolysis Bullosa Simplex. British Journal of Dermatology, 143, 1342-1343.

https://doi.org/10.1046/j.1365-2133.2000.03929.x

[14] Maman, E., Maor, E. and Kachko, L. (1998) Epidermolysis Bullosa, Pyloric Atresia, Aplasia Cutis Congenita: Histopathological Delineation of an Autosomal Recessive Disease. American Journal of Medical Genetics, 78, 127-133.

https://doi.org/10.1002/(SICI)1096-8628(19980630)78:2<127::AID-AJMG6>3.0.CO; $\underline{2-\mathrm{L}}$

[15] Jones, E.M., Hersh, J.H. and Yusk, J.W. (1992) Aplasia Cutis Congenita, Cleft Palate, Epidermolysis Bullosa, and Ectrodactyly: A New Syndrome? Pediatric Dermatology, 9, 293-297. https://doi.org/10.1111/j.1525-1470.1992.tb00351.x

[16] Fine, J.D., Johnson, L.B. and Weiner, M. (2008) Cause-Specific Risks of Childhood Death in Inherited Epidermolysis Bullosa. The Journal of Pediatrics, 152, 276-280. https://doi.org/10.1016/j.jpeds.2007.06.039

[17] Vázquez López, M.E., Fernandez Díaz, M.L. and Somoza Rubio, C. (1998) Aplasia Cutis Congenita, Epidermolysis Bullosa and Ungual Dystrophy. Anales Españoles de Pediatría, 49, 294-296.

[18] Vivona, G., Frontali, M. and Di Nunzio, M.L. (1987) Aplasia Cutis Congenita and/or Epidermolysis Bullosa. American Journal of Medical Genetics, 26, 497-502. https://doi.org/10.1002/ajmg.1320260229

[19] Ozek, C., Gurler, T. and Alper, M. (1999) Aplasia Cutis Congenita with Epidermolysis Bullosa: A Case Report. Annals of Plastic Surgery, 43, 318-320. https://doi.org/10.1097/00000637-199909000-00018

[20] Lim, S.Y., Ho, N.K. and Tan, K.C. (1990) Aplasia Cutis Congenita. A Case Report and Annotation. The Journal of the Singapore Paediatric Society, 32, 164-168. 